\title{
A joint production and transportation planning problem with heterogeneous vehicles
}

\author{
A Toptal ${ }^{* 1}, \mathrm{U} \mathrm{Koc}^{1}$ and I Sabuncuoglu ${ }^{2}$ \\ ${ }^{1}$ Bilkent University, Ankara, Turkey; and ${ }^{2}$ Abdullah Gul University, Kayseri, Turkey
}

\begin{abstract}
We consider a manufacturer's planning problem to schedule order production and transportation to respective destinations. The manufacturer in this setting can use two vehicle types for outbound shipments. The first type is available in unlimited numbers. The availability of the second type, which is less expensive, changes over time. Motivated by some industry practices, we present formulations for three different solution approaches: the myopic solution, the hierarchical solution and the coordinated solution. These approaches vary in how the underlying production and transportation subproblems are solved, that is, sequentially versus jointly or heuristically versus optimally. We provide intractability proofs or polynomial-time exact solution procedures for the sub-problems and their special cases. We also compare the three solution approaches over a numerical study to quantify the savings from integration and explicit consideration of transportation availabilities. Our analytical and numerical results set a foundation and a need for a heuristic to solve the integrated problem. We thus propose a tabu search heuristic, which quickly generates near-optimal solutions.
\end{abstract}

Journal of the Operational Research Society (2014) 65, 180-196. doi:10.1057/jors.2012.184

Published online 27 February 2013

Keywords: production and outbound transportation planning; supply chain management; cargo capacity; hierarchical solution; integrated solution; tabu search

\section{Introduction and related literature}

Transportation of finished goods to customers is an important logistical activity companies must plan for, along with production and inventory management. In traditional supply-chain research and in many industries, planning activities revolve around production, and transportation decisions typically follow production and inventory decisions. However, a growing body of research emphasizes the importance of making these decisions in an integrated manner, particularly accounting for transportation issues (vehicle routing, cost, delivery time, etc) at earlier stages of production planning, to reduce overall costs and increase service levels (Sarmiento and Nagi, 1999; Hall and Potts, 2003; Dawande et al, 2006; Chen, 2010). In keeping with this trend, we consider the production scheduling problem of a company that faces varying vehicle availabilities and costs over time for outbound shipments. We propose mathematical formulations representing different decisionmaking approaches (ie, sequential versus integrated, optimal versus heuristic) and compare their solutions in terms of overall costs.

In the system under consideration, a manufacturer must schedule a certain number of orders. Job processing must be completed by deadline to ensure on-time delivery. Jobs

\footnotetext{
${ }^{*}$ Correspondence: A Toptal, Industrial Engineering Department, Bilkent
} University, Ankara 06800, Turkey. can be delivered to the customers as soon as the order is ready; holding costs are incurred for items that stay in the inventory. Deliveries can be made using a combination of heterogenous vehicles. There are two vehicle types, which differ in their availability and costs over time. An order destined to a specific customer cannot be delivered in multiple batches, and orders for different customers cannot be delivered in the same vehicle. We study the manufacturer's production planning problem in this setting to minimize total inventory holding and outbound transportation costs.

The problem as described above originates from a realworld application. Therefore, in the following sub-section, we begin with a description of the practical setting that motivated our study and a discussion of the extent to which our paper captures the actual practice. We also provide some thoughts on the applicability of the model and the analysis to other settings.

\subsection{Description of the practical motivation}

The problem analysed in the paper is motivated by the practice of a major global appliance manufacturer. This company owns several plants around a certain country, each dedicated to manufacturing a specific family of products (eg, a dishwasher plant, a cooking-appliances plant, a refrigerator plant). The company has domestic and 
international customers. International orders, which constitute the largest portion of sales, have deadlines, whereas domestic orders have due dates. Decisions regarding the acceptance of orders are made ahead of time by the company's sales department for all plants, considering their production capacities. The order information for the amount to be produced in a certain month and their due dates/deadlines are sent by the sales department to each plant about 3 weeks before the start of the month. Plants then make their own detailed production planning decisions. Our paper deals with the type of planning problem that a plant faces in this setting.

A characteristic of the appliance manufacturing industry is that many of the same raw materials are used in all products or in a family of products, for example, steel sheets, plastic and packaging material, among many others. As the consumption rates of these materials are quite independent of the model specifications for actual orders, their replenishment decisions can be made in advance, according to sales forecasts of aggregate units. For example, the company mentioned above imports plastic from outside of the country. Because plastic is highly consumed by all plants, and there are significant set-up costs for ordering, it is purchased in bulk and stored in a central warehouse. The replenishment of plastic by individual plants is then made according to an inventory policy, which often results in periodic, frequent replenishments from the central warehouse. The ownership of the amount of plastic ordered passes to a plant as soon as the material is loaded, and the plant is held accountable for the transportation- and production/ inventory-related costs thereafter. It is because of this predetermined replenishment policy for many materials that a plant has prior information about the arrival times of some containers/vehicles used for inbound shipments before monthly production planning decisions must be made.

The company relies on the services of a few third-party logistics (3PL) providers for all shipments. Three types of containers are commonly used, irrespective of the 3PL provider: $20^{\prime}, 40^{\prime}$ and $40^{\prime}$ high-cube containers. Forty-foot containers are the most frequently used and specifically to deliver international orders. In shipping internationally, multi-modal transportation in the form of land-sea-land usually occurs. In fact, one aspect of international orders is the necessity of containers being at the harbour before a ship's scheduled departure time. Plants incur the costs of first-phase transportation, that is, shipping containers via truck trailers to the harbour. Another characteristic of the majority of international deliveries is that orders for different customers cannot be consolidated within the same container and an order cannot be delivered in multiple batches over time. In contrast, domestic orders can be consolidated and/or split.
In delivering the finished goods to the customers or to the harbour, plants may utilize newly hired vehicles and/or arrange for extended use of incoming vehicles that have been already hired for inbound shipments. With the latter option, an additional fee is paid in proportion to the extended usage time of a vehicle. Using an already hired vehicle may be less costly than hiring a new one, depending on the extra time. There is no limit to the number of vehicles that can be hired; however, the number of incoming vehicles is limited and changes over time. A plant decides the composition of vehicles to be used for each delivery, given the production plan and arrival times of incoming vehicles.

Motivated by the above real-life setting, we focus on a simplified version of a plant's production and outbound transportation planning problem. We consider a setting where all orders have deadlines, different orders cannot be shipped in the same vehicle and an order cannot be delivered in multiple batches. Note that in the real setting, a plant may have a combination of orders with different delivery characteristics or different restrictions on delivery dates, some with due dates and others with deadlines. We also assume that all vehicles have the same capacity, whereas in practice, vehicles with different capacities are available. These aspects of our study better relate to the plant's international orders, which constitute a major portion of sales for the appliance manufacturer. Although our analysis in this paper considers a scenario in which different orders cannot be consolidated, we discuss in Section 7 how the analytical development herein can be extended to the case of consolidation.

The main characteristic common to the manufacturer in our study and a plant in the example is that arrival times of some inbound vehicles are known a priori and can be taken as an input to the planning problem. It is important to note that incoming vehicle schedules may be available before a detailed production plan is made for reasons other than the above noted inventory replenishment policies, for example, purchasing commitments made in advance to take advantage of discounts, or inbound replenishment schedules enforced by suppliers. In the rest of the paper, for the purpose of generality, we simply assume that there are two vehicle types that differ in their availability and costs. The first type of vehicle is available in unlimited numbers, whereas the second type, which is less costly, has limited and time-varying availability. Because the heterogeneity of outbound vehicles as modelled herein may be encountered in practice for reasons other than incoming vehicles (eg, different vehicle types offered by different 3PL providers or the pricing strategy of a single $3 \mathrm{PL}$ provider), we do not specifically refer to the second type of vehicle as an incoming vehicle.

In the next subsection, we discuss the theoretical and practical contributions of the paper in relation to the literature. 


\subsection{Contributions of the paper in relation to the literature}

As reported in many recent papers on supply chain scheduling (eg, Chen and Vairaktarakis, 2005; Wang and Lee, 2005; Chen, 2010), and evidenced in our relations with this manufacturer as well with others, we concluded that it is common industry practice that outbound transportation decisions (eg, transport mode choice, vehicle scheduling and routing) are made, following a production plan. Furthermore, as objectives related to production and customer service are given more priority, transportation costs are either ignored or considered too late in the process to determine a less costly delivery plan. We have identified three solution approaches regarding the decisionmaking process for planning order production and outbound transportation: the myopic solution, the hierarchical solution and the coordinated solution. In the myopic and the hierarchical solutions, production planning decisions are made first, followed by outbound transportation decisions. In the myopic solution, planning efforts for transportation are limited, often made using a heuristic and without giving explicit consideration to transportation costs and constraints. In the hierarchical solution, transportation planning is done in more detail in an effort to optimize the related costs. Finally, in the coordinated solution, production and transportation decisions are made jointly, aiming to minimize overall costs. Explanations of these solution approaches within the specific context of our problem will be provided in Section 3.

We first present mathematical formulations for solving the problem using the three approaches. The formulations for the myopic and hierarchical solutions are based on identifying two subproblems: the production subproblem and the transportation subproblem. In the production subproblem, the objective is to find a schedule of jobs to minimize inventory holding costs with no job being tardy. In the transportation subproblem, a plan is made to deliver completed orders with the least cost considering the different vehicle availabilities. We show that solving the production subproblem is NP-hard in the strong sense; however, we come up with polynomial algorithms for solving the two subproblems, given the delivery times of orders. This problem structure enables us to propose a novel application of the tabu search method as a heuristic to minimize the sum of inventory holding and transportation costs.

This paper is related to the literature on integrated production and transportation planning. Examples of studies in this area are Chang and Lee (2004), Chen and Vairaktarakis (2005), Chen and Pundoor (2006), Li and Ou (2005), Li and Vairaktarakis (2007), Geismar et al (2008), Wang and Cheng (2009) and Geismar et al (2011). Chen (2010) provides a review of this literature, focusing on outbound transportation issues. Few papers account for inbound and outbound transportation simultaneously (ie, Li and Ou, 2005; Wang and Cheng, 2009), and our study, like many (eg, Chang and Lee, 2004; Chen and Vairaktarakis, 2005; Li et al, 2005; Li and Vairaktarakis, 2007; Zhong et al, 2007), concentrates on outbound transportation. Some distinguishing features of our study include modelling heterogenous vehicle types, the objective function being considered and quantifying the differences between various solution approaches.

It is important to note that a majority of the papers on integrated production and transportation planning model the existence of a single mode of transportation (eg, Chang and Lee, 2004; Chen and Vairaktarakis, 2005; Li et al, 2005; Wang and Cheng, 2007; Geismar et al, 2011). Wang and Lee (2005), Lei et al (2006), Stecke and Zhao (2007) and Chen and Lee (2008) are examples of the few studies that account for different transportation choices. However, in most of these studies (eg, Wang and Lee, 2005; Stecke and Zhao, 2007; Chen and Lee, 2008), the difference among the transportation choices stems from delivery time and cost. It is assumed that the mode of transportation with a shorter delivery time is more costly. Transportation costs are part of the objective function, and delivery times of orders either contribute to the costs (see Chen and Lee, 2008, and the second problem in Wang and Lee, 2005) or they are incorporated in a constraint allowing for no tardiness (see Stecke and Zhao, 2007 and the first problem in Wang and Lee, 2005). In our study, vehicle costs and capacities are explicitly modelled, and vehicles are considered as heterogenous owing to the differences in their costs and availabilities. Mainly, the less costly vehicle is less available. Furthermore, we take minimization of inventory holding and transportation costs as an objective and do not allow for any job to be tardy.

A group of studies within the general area of integrated production and transportation planning considers coordinating the production, inventory and transportation routing operations simultaneously (eg, Chandra and Fisher, 1994; Lei et al, 2006; Bard and Nananukul, 2009; Bard and Nananukul, 2010). These studies consider inventory holding costs at all locations (including the customer locations), and routing vehicles among geographically dispersed customers is part of the problem. Another common property of these papers is that delivering portions of an order at multiple points in time and consolidating different orders are allowed. Our model does not consider inventory holding costs at customer locations nor vehicle routing. However, orders that cannot be split and consolidated must be assigned to heterogenous vehicles with the characteristics mentioned previously, and this makes our problem challenging.

While it is obvious that integrating production scheduling and transportation decisions reduces total costs, as opposed to making the related decisions in a sequential manner, the benefits of integration are not well studied. We have identified few papers (Chandra and Fisher, 1994; 
Chen and Vairaktarakis, 2005; Pundoor and Chen, 2005; Dawande et al, 2006) investigating this issue within the contexts of their problem settings. By comparing our hierarchical solution with our coordinated solution, we quantify the savings because of integration, and similar to the results of these studies, we conclude that these savings can in fact be significant. Note that all of these studies assume homogenous vehicles, and therefore allocating deliveries among different types of vehicles is not an issue. Furthermore, different from the reviewed papers, by comparing our myopic solution with our hierarchical solution, we quantify the savings that can be achieved by optimal usage of the transportation choices. Our detailed analysis of the underlying subproblems not only helps us in making these comparisons, but our proposed tabu search heuristic for the coordinated problem also relies on the solutions of these subproblems. Chandra and Fisher (1994) utilize a similar approach for a different setting, but the transportation subproblem in their paper is a multiperiod vehicle-routing problem and is solved heuristically, whereas in ours it is solved optimally. In addition, the procedure they propose to solve the integrated problem is a local improvement heuristic.

It is worthwhile to note that in comparing the different solution approaches, our purpose is not to promote one approach over another; there may be practical needs or issues other than costs that companies consider when choosing among different approaches. For example, the myopic and the hierarchical solutions do not require that the information regarding transportation availabilities is known at the time that production decisions are made. In fact, to make a transportation decision for a period, the myopic solution uses only the information that is relevant to that period. The hierarchical solution is based on the premise that information regarding transportation availabilities in all periods is known at the beginning of the planning period. In situations where this information becomes available at a cost, companies should weigh the benefits of different approaches against the cost of information. We note that the value of information sharing between different parties in the supply chain, specifically a retailer's sharing demand information with his/her manufacturer, has been reported in the literature (eg, Lee $e t$ al, 2000; $\mathrm{Li}, 2002$ ). The results of our paper indicate that a manufacturer may achieve significant savings if a carrier shares information about his/her vehicle availabilities at the time that the manufacturer makes his/her production planning decisions.

In the next section, we begin with a detailed description of the problem and the notation. We continue in Section 3 with the explanation and the modelling of the three solution approaches. In Section 4, we provide a further analysis of the underlying subproblems. In Section 5, we propose a heuristic based on the tabu search for the joint problem of minimizing inventory holding and transportation costs.
This is followed by the results of an extensive numerical analysis comparing the three solution approaches and the performance of the heuristic. Section 7 concludes the paper.

\section{Problem definition and notation}

We consider a manufacturer's production planning and delivery scheduling problem, which concerns $N$ orders to be satisfied in $T$ periods. The manufacturer's production capacity is limited by $P_{t}$ units in period $t$, indepedent of the type of items to be produced. The production for each order $i$, which has size $S_{i}$, must be completed and the order must be delivered before its deadline $D_{i}$. Late deliveries are not allowed. In this setting, order acceptance and rejection decisions have been already made, and there exists a feasible production plan that ensures every order is ready for delivery before its deadline. The cost of carrying one unit of inventory from one period to the next amounts to $\$ H$ for all orders.

Orders are delivered to customers at the expense of the manufacturer. The manufacturer uses capacitated vehicles for outbound transportation. Each vehicle holds up to $K$ units of the finished product. Any number of these vehicles can be utilized at a cost of $\$ C_{1}$ per vehicle in each period. However, in period $t$, a limited number (ie, $A_{t}$ ) of vehicles is also available at a lower cost (ie, $C_{2}$ ). In the rest of the text, we will refer to the vehicles with cost $C_{1}$ as type $I$ and to those with cost $C_{2}$ as type II. The latter type of vehicle can be held at the facility at an additional cost of $\$ W$ per vehicle per period. The following restrictions exist on outbound shipments: (i) customers do not accept partial deliveries, and (ii) different orders cannot be shipped in the same vehicle. Therefore, the number of vehicles needed for delivery of order $i$ is given by $\left\lceil S_{i} / K\right\rceil$. The problem is to find a production plan that minimizes the sum of transportation and inventory holding costs. The plan must imply the order delivery schedule, the number of both types of vehicles used in outbound transportation and the production quantity in each period. Different approaches may be used to solve the production planning problem in this setting. Before proceeding with a detailed discussion of these approaches in the next section, we summarize below some of the notations used in the paper. Additional notations will be defined when necessary.

$N \quad$ Number of orders.

$T \quad$ Number of periods.

$P_{t} \quad$ Production capacity in period $t$.

$S_{i} \quad$ Size of order $i$.

$D_{i} \quad$ Deadline of order $i$

$H$ Cost of carrying one unit of inventory from one period to the next.

$K \quad$ Capacity of a truck in number of units.

$C_{1} \quad$ Cost of utilizing a type I vehicle. 
$C_{2} \quad$ Cost of utilizing a type II vehicle.

$W \quad$ Cost of holding a type II vehicle for a period.

$A_{t} \quad$ Number of type II vehicles available in period $t$.

Cost $_{m}$ Total cost of the myopic solution.

Cost $_{h}$ Total cost of the hierarchical solution.

Cost $t_{c}$ Total cost of the coordinated solution.

We would like to emphasize that the availability of type II vehicles during the planning horizon (ie, $A_{t}$ ) is exogenously given and is an input to the model.

\section{Solution approaches}

In this section, we discuss the three approaches briefly introduced in Section 1 (coordinated solution, myopic solution and hierarchical solution) for solving the problem of interest. In the coordinated solution, production and transportation decisions are made jointly in a single step. The other two approaches follow a two-step process, which relies on sequentially solving the underlying subproblems of production and transportation. The production subproblem is to, in each period, find the production quantity and the order delivery schedule to minimize inventory holding costs. Because this problem is solved independently, without considering outbound shipment costs, its optimal solution does not foresee savings from transportation costs if the completed orders are held in the inventory. Therefore, a plan that minimizes inventory holding costs delivers the orders as soon as they are completed. The transportation subproblem is, given the delivery schedule of orders, to determine the number of type I and type II vehicles to be used over time to minimize transportation costs. Note that in this setting, production and transportation decisions interact for the following two reasons: first, the shipment costs of an order may be different in different periods because of the time-varying availability of the type II vehicles, and an order can be shipped only when its production is completed; second, there is a trade-off between increasing the inventory holding costs of completed items and carrying them over periods to take advantage of less costly transportation alternatives in the future.

The first steps of the myopic and the hierarchical solutions are the same, and mainly solve the production subproblem optimally. The two solutions differ in their second step, where the transportation subproblem is solved. In the hierarchical solution, this subproblem is also solved optimally, whereas in the myopic solution it is not. More specifically, in the myopic solution, transportation arrangements are made to deliver the completed orders in each period using only the vehicles available in that period. As type II vehicles are less costly, they are preferred over type I vehicles. If there is no type II vehicle, outbound shipments are made using type I vehicles. As an implication of this difference, the hierarchical solution allows for holding type II vehicles over periods to satisfy future delivery requirements, while the myopic solution does not.

In the remaining parts of this section, we present these approaches in more detail. The following is a list of decision variables common to all three approaches:

$\pi_{t} \quad$ Total production amount in period $t \quad t=1, \ldots, T$

$I_{t} \quad$ Inventory carried from period $t$

to $t+1$

$y_{t} \quad$ Number of type I vehicles used

in period $t$

$x_{t} \quad$ Number of type II vehicles used in period $t$

$w_{t} \quad$ Number of type II vehicles carried

from period $t$ to $t+1$$$
t=1, \ldots, T
$$

$t=1, \ldots, T$

$t=1, \ldots, T$

$t=1, \ldots, T$

$t=1, \ldots, T$

$\sigma_{t i}\left\{\begin{array}{lll}1 & \text { If order } i \text { is delivered in period } t & t=1, \ldots, T ; \\ 0 & \text { otherwise } & i=1, \ldots, N\end{array}\right.$

\subsection{Coordinated solution}

In presenting the details of the different solution approaches, we begin with the coordinated solution. The following integer programming formulation models all aspects of outbound transportation to obtain a production plan. We refer to this model as the Integrated Model and its optimal objective function value is for a problem instance using the notation Cost $_{c}$.

Integrated Model:

$$
\begin{gathered}
\text { Min } \sum_{t=1}^{T}\left(C_{2} x_{t}+C_{1} y_{t}+W w_{t}+H I_{t}\right) \\
I_{t}=I_{t-1}+\pi_{t}-\sum_{i=1}^{N} \sigma_{t i} S_{i} \quad t=1, \ldots, T \\
x_{t} \leqslant A_{t}+w_{t-1}-w_{t} \quad t=1, \ldots, T \\
\pi_{t} \leqslant P_{t} \quad t=1, \ldots, T \\
\sum_{i=1}^{N}\left\lceil S_{i} / K\right\rceil \sigma_{t i}=x_{t}+y_{t} \quad t=1, \ldots, T \\
\sum_{t=1}^{D_{i}} \sigma_{t i}=1 \quad i=1, \ldots, N \\
x_{t}, y_{t}, w_{t}, \pi_{t}, I_{t} \in\{0\} \cup Z^{+} \quad t=1, \ldots, T \\
\sigma_{t i} \in\{0,1\} \quad t=1, \ldots, T ; i=1, \ldots, N \\
w_{0}=0, I_{0}=0
\end{gathered}
$$

The objective function in the above formulation is the sum of the transportation and inventory holding costs. The first constraint set represents the inventory balance equations. Inequality (2) signifies that the number of type II vehicles needed in period $t$ cannot exceed the number that is available in period $t$. The type II vehicles needed 
in period $t$ include those used for the shipments in period $t$ (ie, $x_{t}$ ) as well as those that are carried to the next period (ie, $w_{t}$ ). The number of type II vehicles available in period $t$ includes those that have been carried from earlier periods (ie, $\left.w_{t-1}\right)$ as well as those that have become recently available in period $t$ (ie, $A_{t}$ ). Constraint set (2) is in the form of an inequality because some of the type II vehicles available in period $t$ may not be utilized at all. Inequality (3) ensures that production capacity is not exceeded in any period. Equation (4) implies that the total demand for vehicles to be used in a period's outbound shipment is satisfied through either type I or type II vehicles. Equation (5) guarantees that every order is delivered before its deadline. Constraint sets (6)-(8) refer to the non-negativity, integrality and initial conditions of some variables, respectively. Here, $Z^{+}$is the set of positive integers.

The mathematical formulation introduced above explicitly considers the transportation costs and capacities in making production planning decisions. Koç et al (2013) show that the problem of interest as modelled herein is NP-hard in the strong sense. In the next section, we present the other two approaches in detail.

\subsection{Other solution approaches: myopic and hierarchical}

Recall that the myopic and hierarchical solutions rely on the production subproblem and the transportation subproblem. The formulations of these subproblems are decomposed from the Integrated Model and presented below.

Production Subproblem:

$$
\begin{array}{rl}
\operatorname{Min} \sum_{t=1}^{T} H I_{t} & \\
I_{t}=I_{t-1}+\pi_{t}-\sum_{i=1}^{N} \sigma_{t i} S_{i} & t=1, \ldots, T \\
\pi_{t} \leqslant P_{t} & t=1, \ldots, T \\
\sum_{t=1}^{D_{i}} \sigma_{t i}=1 & i=1, \ldots, N \\
\pi_{t}, I_{t} \in\{0\} \cup Z^{+} & t=1, \ldots, T \\
I_{0}=0 & \\
\sigma_{t i} \in\{0,1\} & t=1, \ldots, T ; \\
& i=1, \ldots, N
\end{array}
$$

Transportation Subproblem:

$$
\begin{array}{rr}
\operatorname{Min} \sum_{t=1}^{T}\left(C_{2} x_{t}+C_{1} y_{t}+W w_{t}\right) & \\
x_{t} \leqslant A_{t}+w_{t-1}-w_{t} & t=1, \ldots, T \\
x_{t}+y_{t}=\sum_{i=1}^{N}\left\lceil S_{i} / K\right\rceil \tilde{\sigma}_{t i} & t=1, \ldots, T \\
x_{t}, y_{t}, w_{t} \in\{0\} \cup Z^{+} & t=1, \ldots, T \\
w_{0}=0 &
\end{array}
$$

In the production subproblem, issues related to transportation are not considered. Similarly, the transportation subproblem does not consider production- and inventoryrelated costs and constraints. Note also that the indicator variable showing whether a delivery is to be made for order $i$ in period $t$, that is, $\sigma_{t i}$, is a decision variable in the production subproblem, whereas its value is an input to the transportation subproblem. In the transportation subproblem, $\tilde{\sigma}_{t i}$ denotes a given value of $\sigma_{t i}$.

Now, we are ready to provide detailed descriptions of the myopic and hierarchical solutions. Before doing so, we define a further piece of notation. Let $\operatorname{Cost}_{p r}$ and $\sigma_{t i}^{*}$ be the optimal values of the objective function and $\sigma_{t i}$, respectively, as an output of the production subproblem. This solution implies that the total vehicle requirement for deliveries in period $t$ is $\sum_{i=1}^{N}\left\lceil S_{i} / K\right\rceil \sigma_{t i}^{*}$. Therefore, the following description applies to the myopic solution.

\section{Description of The Myopic Solution:}

1. Solve the production subproblem.

2. Set $x_{t}=\min \left(\sum_{i=1}^{N}\left\lceil S_{i} / K\right\rceil \sigma_{t i}^{*}, A_{t}\right), y_{t}=\sum_{i=1}^{N}\left\lceil S_{i} / K\right\rceil \sigma_{t i}^{*}-x_{t}$ and $w_{t}=0$. Compute the resulting costs as follows:

$$
\operatorname{Cost}_{m}=\operatorname{Cost}_{p r}+\sum_{t=1}^{T}\left(C_{2} x_{t}+C_{1} y_{t}\right)
$$

In comparison to the myopic solution, the second step of the hierarchical solution exploits the possibility of carrying type II vehicles from one period to the next to take better advantage of the cheaper transportation alternative. More specifically, holding a type II vehicle for a delivery that must take place within the next $\beta$ periods is less costly than using a type I vehicle for the same delivery, where

$$
\beta=\left\lceil\frac{C_{1}-C_{2}}{W}\right\rceil-1 \text {. }
$$

As it will be discussed in Section 4, the value of $\beta$ is critical as an input to our proposed algorithm for the optimal solution of the transportation subproblem. Therefore, it is also utilized by the following algorithm for obtaining the hierarchical solution and the resulting cost.

\section{Description of the Hierarchical Solution:}

1. Solve the production subproblem and do the following initialization of variables:

(a) For $t=1$ to $t=\mathrm{T}$ and for $i=1$ to $i=N$, set $\tilde{\sigma}_{t i}=\sigma_{t i}^{*}$.

(b) Compute the value of $\beta$ using Expression (9).

2. Solve the transportation subproblem given $\tilde{\sigma}_{t i}$ and $\beta$. Compute Cost $_{h}$ as the summation of the optimal costs of the two subproblems (ie, $\operatorname{Cost}_{h}=\operatorname{Cost}_{p r}+\operatorname{Cost}_{t r}\left(\tilde{\sigma}_{t i}\right)$ ).

Here, $\operatorname{Cost}_{t r}\left(\tilde{\sigma}_{t i}\right)$ refers to the optimal objective function value of the transportation subproblem, given the delivery dates of orders as implied by the optimal solution of the production subproblem. 
The second step of the hierarchical solution is essentially solving the transportation subproblem optimally, given the optimal solution to the production subproblem. We note that the transportation subproblem may be solved without using the specific definition of $\beta$. How we use $\beta$ in solving the transportation subproblem will become clearer when we present a polynomial-time exact solution procedure in the next section.

\section{Analysis of the subproblems}

Recall from Section 3.2 that both the myopic and hierarchical approaches utilize the solution of the production subproblem, and the hierarchical approach further requires the solution of the transportation subproblem. In addition, the tabu search heuristic that will be described in Section 5 is based on solving these two subproblems optimally for given delivery dates. Therefore, we analyse them further in this section. We start with establishing the status of the production subproblem in the next theorem. Thereafter, we present polynomial time algorithms for obtaining optimal solutions of the two subproblems, given the order delivery dates. How the delivery dates are determined and updated through the steps of the tabu search heuristic will be discussed in Section 5 .

Theorem 1 The production subproblem (production planning problem without transportation considerations) is NP-hard in the strong sense

Proof The proof is done by a reduction from the 3-Partition (3P) problem. Note that the production planning problem without transportation considerations is clearly in NP. The 3P problem is defined as follows:

INSTANCE: Set $\mathcal{G}$ of $3 t$ elements, a bound $B \in Z^{+}$and a size $s(a) \in Z^{+}$for each $a \in \mathcal{G}$ such that $B / 4<s(a)<B / 2$ and such that $\sum_{a \in \mathcal{G}} s(a)=t B$.

QUESTION: Can $\mathcal{G}$ be partitioned into $t$ disjoint sets $\mathcal{G}_{1}, \mathcal{G}_{2}, \ldots, \mathcal{G}_{t}$ such that $\sum_{a \in \mathcal{G}_{\tau}} s(a)=B$ for $\tau=1,2, \ldots, t$ (note that each $\mathcal{G}_{\tau}$ must therefore contain exactly three elements from $\mathcal{G})$ ?

REDUCTION: Take an arbitrary instance of 3P. The corresponding instance of our problem is constructed as follows: For each element $a$ in set $\mathcal{G}$ define an order with size $S_{a}=s(a)$ (ie, $\left.N=\|\mathcal{G}\|\right)$. Set $T=t, H=1$ and $P_{t}=B$, and, for each $a=1,2, \ldots, N$ set $D_{a}=T$. We will show that there is a solution to $3 \mathrm{P}$ if there is a solution to our problem with cost less than or equal to $z^{*}=0$.

Assume that there is a solution to our problem with cost $z$ that is less than or equal to 0 . Thus, no inventory holding cost is incurred. As there are $3 t$ orders to be satisfied with a total size of $t B$ and total production capacity of the facility is equal to total demand $\left(\sum_{t=1}^{T} P_{t}=t B\right)$, the total number of items produced at each period is equal to $B$. This means that three orders with total size equal to $P$ are completed and delivered at each period, with no inventory held at the facility. Now construct a solution to $3 \mathrm{P}$ as follows: For all orders produced and delivered in period $\tau$, put the corresponding element in set $\mathcal{G}$ into $\mathcal{G}_{\tau}$. As the size of orders $S_{a}=s(a), \quad$ for each disjoint set $\mathcal{G}_{\tau}, \quad \sum_{a \in \mathcal{G}_{\tau}} s(a)=B$
$(\tau=1,2, \ldots, t)$.

If there is a solution to $3 \mathrm{P}$, construct a solution to our problem instance as follows: For each disjoint set $\mathcal{G}_{\tau}$, $\tau=1,2, \ldots, t$, produce and deliver all the items of order $a \in \mathcal{G}_{\tau}$ in period $\tau$. A similar reduction with the previous case implies that the solution has a cost of $z=0$.

Now, let us consider the two subproblems, given the delivery dates of all orders. Note that it is always possible to obtain a feasible solution to the transportation subproblem simply by using the type I vehicles, which are abundant. The production subproblem, on the other hand, may not be feasible depending on the delivery dates given. More specifically, if the total size of orders that must be completed and sent by time $t$ is greater than the cumulative production capacity until that period, the production subproblem is infeasible. We propose the following algorithm for finding an optimal solution to the production subproblem, given that $\sigma_{t i}=\tilde{\sigma}_{t i}$. With a slight change of notation, $\operatorname{Cost}_{p r}\left(\tilde{\sigma}_{t i}\right)$ is used to refer to the optimal costs of the production subproblem under the given order delivery dates.

Algorithm I Optimal solution of the production subproblem given the delivery dates:

1. Do the following initialization of variables:

(a) Set $\operatorname{Cost}_{p r}\left(\tilde{\sigma}_{t i}\right)=0$.

(b) For $t=1$ to $t=T$, set $F_{t}=\sum_{i=1}^{N} \tilde{\sigma}_{t i} S_{i}$.

2. For $t=T$ down to $t=1$ :

(a) Determine the production amount in period $t$ using $\pi_{t}=\min \left\{F_{t}, P_{t}\right\}$

(b) If $F_{t}>\pi_{t}$,

(i) If $t=1$, then there is no feasible solution. Stop and exit.

(ii) If $t \neq 1$, do the following:

A. $F_{t-1}=F_{t-1}+F_{t}-\pi_{t}$.

B. Update the optimal costs using $\operatorname{Cost}_{p r}\left(\tilde{\sigma}_{t i}\right)=$ $\operatorname{Cost}_{p r}\left(\tilde{\sigma}_{t i}\right)+\left(F_{t}-\pi_{t}\right) \times H$.

In the above algorithm, $F_{t}$ is the amount that must be produced within $[1, t]$ for the deliveries that will take place within $[t, T]$. The algorithm follows a backward recursive path to find the production quantity in each period and the 
resulting cost. It can be observed that the above algorithm runs in $O(T)$.

Proposition 1 Algorithm I terminates with an optimal solution for the production subproblem if the given delivery dates are feasible, otherwise it returns no solution.

Proof Let us consider the last period for a problem with $T$ number of periods. $\sum_{i=1}^{N} \tilde{\sigma}_{T i} S_{i}$ is the total size of orders that are to be delivered in the last period. As many as possible of these items should be produced in the last period, because doing otherwise increases inventory holding costs. Therefore, $\pi_{T}=\min \left\{\sum_{i=1}^{N} \tilde{\sigma}_{T i} S_{i}, P_{T}\right\}$ is the amount to be produced in period $T$, as given by Step 2(a) of the algorithm. If $T=1$ and $\sum_{i=1}^{N} \tilde{\sigma}_{1 i} S_{i}>P_{1}$, then the production capacity in the whole planning horizon is not enough to satisfy the demand, and therefore there is no feasible solution. In this case, the algorithm terminates in Step 2(b).i with no solution. If $T=1$ and $\sum_{i=1}^{N} \tilde{\sigma}_{1 i} S_{i} \leqslant P_{1}$, then $\pi_{1}=\min \left\{\sum_{i=1}^{N} \tilde{\sigma}_{1 i} S_{i}, P_{1}\right\}$ is the optimal solution, and is given by Step 2(a).

If $\sum_{i=1}^{N} \tilde{\sigma}_{T i} S_{i}>P_{T}$ and $T \geqslant 2$, then the production capacity in the last period is not enough to satisfy the demand of that period, therefore $F_{T}-P_{T}=\sum_{i=1}^{N} \tilde{\sigma}_{T i} S_{i}-$ $P_{T}$ of this amount should have been produced and carried as inventory from earlier periods. Therefore, $F_{T-1}$, which is initially set to $\sum_{i=1}^{N} \tilde{\sigma}_{T-1, i} S_{i}$, is increased by $F_{T}-P_{T}$ in Step 2(b).ii.A of the algorithm. Because at this point we know that $F_{T}-P_{T}$ number of items will be held in the inventory for at least one period, the total costs are updated in Step 2(b).ii.B to incorporate the cost of holding this much inventory for a period. If $\sum_{i=1}^{N} \tilde{\sigma}_{T i} S_{i} \leqslant P_{T}$ and $T \geqslant 2$, then the production capacity in the last period is enough to satisfy the demand in the last period, therefore no inventory should be carried from earlier periods to the last period, and hence $F_{T-1}$ stays the same as its initial value $\sum_{i=1}^{N} \tilde{\sigma}_{T-1, i} S_{i}$.

The algorithm next finds the quantity to be produced in period $T-1$ (ie, $\pi_{T-1}$ ), and updates $F_{t-2}$. Continuing in this manner, the algorithm finally arrives at the first period. $F_{1}$ is the amount to be produced in period 1 for a feasible solution to be obtained. If $P_{1} \geqslant F_{1}$, then the algorithm sets $\pi_{1}=F_{1}$ as the optimal production amount in period 1 . If $P_{1}<F_{1}$, then, because there are no more production periods to supply the requirements, the algorithm exits with no solution in Step 2(b).i.

The following algorithm solves the transportation subproblem optimally for the given delivery dates $\tilde{\sigma}_{t i}$ :

\section{Algorithm II Optimal solution of the transportation} subproblem:

1. Do the following initialization of variables: (a) Set $\operatorname{Cost}_{t r}\left(\tilde{\sigma}_{t i}\right)=0$.

(b) For $t=1$ to $t=T$, set $x_{t}=0, w_{t}=0, G_{t}=$ $\sum_{i=1}^{N}\left\lceil S_{i} / K\right\rceil \tilde{\sigma}_{t i}$ and $\varphi_{t}=A_{t}$.

2. For $b=0$ to $\beta$ : For $t=1$ to $T-b$ :

(a) Determine the number of type II vehicles among those that become available in period $t$ to be used in period $t+b$. That is, compute $v_{t}=\min \left\{G_{t+b}, \varphi_{t}\right\}$.

(b) Update the number of vehicles needed for deliveries in period $t+b$ using $G_{t+b}=G_{t+b}-v_{t}$.

(c) Decrease the number of type II vehicles available in period $t$ by $v_{t}$ (that is, set $\varphi_{t}=\varphi_{t}-v_{t}$ ).

(d) Increase the number of type II vehicles utilized in period $t+b$ by $v_{t}$ (that is, set $x_{t+b}=x_{t+b}+v_{t}$ ).

(e) If $b>0$, for $\tau=0$ to $(b-1)$ set $w_{t+\tau}=w_{t+\tau}+v_{t}$.

3. For $t=1$ to $t=T$,

(a) Set $y_{t}=G_{t}$.

(b) Update the optimal costs using $\operatorname{Cost}_{t r}\left(\tilde{\sigma}_{t i}\right)=$ $\operatorname{Cost}_{t r}\left(\tilde{\sigma}_{t i}\right)+w_{t} \times W+x_{t} \times C_{2}+y_{t} \times C_{1}$.

In the above algorithm, $b$ represents the number of periods for which a type II vehicle is held. Expression (9) implies that it is not optimal to hold a type II vehicle for more than $\beta$ number of periods. Therefore, $b$ ranges from 0 to $\beta$. Within steps 2(a)-2(e) of the algorithm, first, among the type II vehicles that have been on hold for the last $b$ periods, the number that will be used in period $t+b$ is found. Later, the overall need for vehicles in period $t+b$ (ie, $G_{t+b}$ ), the number of type II vehicles available in period $t$ (ie, $\left.\varphi_{t}\right)$, the number of type II vehicles used in period $t+b$ (ie, $x_{t+b}$ ) and the inventory of vehicles throughout periods $t$ to $t+b-1$ (ie, $w_{t+\tau}$ for $\tau=0, \ldots$, $b-1)$ are updated. The algorithm runs steps 2(a)-2(e) in such a sequence of $t$ and $b$ values that type II vehicles are used in the most immediate period that a need for vehicles arises. In this way, the holding cost of vehicles is minimized, along with the total transportation costs. In the last step of the algorithm, a plan is made to satisfy the remaining need for vehicles in any period using type I vehicles, and the cost is updated. We note that the time complexity of this algorithm is $O\left(T \beta^{2}\right)$, which is bounded by $O\left(T^{3}\right)$.

Proposition 2 Algorithm II terminates with an optimal solution for the transportation subproblem.

Proof If $\beta=0$, then $C_{1} \leqslant C_{2}+W$, which implies that it is never optimal to carry a type II vehicle over periods. As $C_{1}>C_{2}$, if a type II vehicle becomes available in period $t$ and a need for a vehicle arises in that period, then the type II vehicle must be utilized. Therefore, $x_{t}=$ $\min \left\{\sum_{i=1}^{N}\left\lceil S_{i} / K\right\rceil \tilde{\sigma}_{t i}, A_{t}\right\}$ for $t=1,2, \ldots, T$. This is given by Steps 2(a) and 2(d) of the algorithm for a certain $t$. If $\sum_{i=1}^{N}\left\lceil S_{i} / K\right\rceil \tilde{\sigma}_{t i}>A_{t}$ for a period $t$, then the vehicle 
requirements cannot be satisfied by only using type II vehicles but an additional $G_{t}=\sum_{i=1}^{N}\left\lceil S_{i} / K\right\rceil \tilde{\sigma}_{t i}-A_{t}$ type I vehicles are needed. If $\sum_{i=1}^{N}\left\lceil S_{i} / K\right\rceil \tilde{\sigma}_{t i} \leqslant A_{t}$, then there is no need for type I vehicles in period $t$ (ie, $\left.G_{t}=0\right)$. The last two statements are ensured by Step 2(b) of the algorithm. At this point, $G_{t}$ gives the number of type I vehicles needed for deliveries in period $t$. Therefore, $y_{t}$ is set to $G_{t}$ in Step 3(a). The transportation cost of this plan is $\sum_{t=1}^{t=T}\left(x_{t} \times C_{2}+y_{t} \times C_{1}\right)$, as given in Step 3(b) of the algorithm.

If $\beta \geqslant 1$, then $C_{2}+b W<C_{1}$ for $1 \leqslant b \leqslant \beta$ because of Expression (9). This implies holding a type II vehicle that becomes available in period $t$ for $b$ periods and utilizing it in period $t+b$, is less costly than using a type I vehicle in period $t+b$, for all $b$ such that $b \leqslant \beta$. Notice that as the planning horizon is $T$ periods, for a given value of $b$, it only makes sense to carry a type II vehicle that becomes available in periods $t=1$ to $t=T-b$ for $b$ periods. For all such possible values of $b$ and $t$, the algorithm first considers the assignment of type II vehicles that become available in period $t$ to deliveries in period $t+b$. Then, if there is still need for vehicles in any period, it is satisfied through type I vehicles in Step 3.

Now, let us show that the assignment of type II vehicles to the deliveries in different periods, which is done in Step 2 of the algorithm, is optimal. In general, type II vehicles must be used in the most immediate period that a need for vehicles arises. Keeping a type II vehicle for future use while there is a need in the current period, would increase the transportation cost by as much as the holding cost of the type II vehicle, and hence would not be optimal. Therefore, the algorithm begins with $b=0$ to satisfy as many of the vehicle requirements as possible in each period through the type II vehicles that become available in that period. As a result, Step 2(a) and Step 2(d) jointly lead to $x_{t}=\min \left\{\sum_{i=1}^{N}\left\lceil S_{i} / K\right\rceil \tilde{\sigma}_{t i}, A_{t}\right\}$. If $\sum_{i=1}^{N}\left\lceil S_{i} / K\right\rceil \tilde{\sigma}_{t i} \leqslant A_{t}$, then all the vehicle requirements in period $t$ are satisfied through type II vehicles that become available in period $t$. There is no further requirement in period $t$, which implies $G_{t}=0$, as given by Step 2(b) of the algorithm. The remaining $\varphi_{t}=A_{t}-\sum_{i=1}^{N}\left\lceil S_{i} / K\right\rceil \tilde{\sigma}_{t i}$ type II vehicles can be used in future periods, as given by Step 2(c) of the algorithm. If $\sum_{i=1}^{N}\left\lceil S_{i} / K\right\rceil \tilde{\sigma}_{t i}>A_{t}$, then all the type II vehicles that become available in period $t$ are used for the deliveries in period $t$, therefore, we have $\varphi_{t}=0$, as given by Step 2(c). However, there is still need for $G_{t}=$ $\sum_{i=1}^{N}\left\lceil S_{i} / K\right\rceil \tilde{\sigma}_{t i}-A_{t}$ additional vehicles in period $t$.

After as many of the type II vehicles as possible are utilized in the period that they become available, the algorithm considers utilization of type II vehicles one period after they become available, that is, $b=1$. Continuing in this manner, Step 2 of the algorithm ends with considering utilization of type II vehicles $\beta$ periods after they become available, that is, $b=\beta$. After this step, if there is still a vehicle requirement in any period $t$ (ie, $G_{t}>0$ ), then $G_{t}$ type I vehicles are used. If there are type II vehicles that are not assigned to the deliveries in any period, they are unused/released.

We conducted an extensive numerical analysis to compare the three solution approaches introduced in Section 3. The results, which are discussed in more detail in Section 6, show that the total costs of the coordinated solution may be less than that of the myopic solution by as much as $75 \%$ and less than that of the hierarchical solution by as much as $58 \%$. Owing to such results derived from the computational analysis, we conclude that significant savings can be achieved if the coordinated solution is used instead of the other two approaches. Furthermore, all three approaches rely on solving problems that are NP-hard in the strong sense. Therefore, the myopic solution and the hierarchical solution do not provide a computational advantage over the coordinated solution. These results establish a need for a heuristic that can be used in practice to make production planning and transportation decisions jointly, as in the coordinated solution. In the next section, we propose a meta-heuristic that utilizes the tabu search technique for this purpose.

\section{Tabu search}

The myopic and hierarchical solutions are based on our observation that production planning decisions are made before transportation decisions in many real-life practices. In these two approaches, first, the production subproblem is solved optimally. Then, transportation is arranged to comply with a production plan that minimizes inventory holding costs. In the hierarchical solution, the transportation subproblem is also solved optimally. As both approaches focus on sequentially minimizing the two cost components, total costs are not necessarily optimized. The tabu search heuristic that we propose is also based on the two subproblems. However, as opposed to the myopic and hierarchical solutions, the tabu search uses the solutions of these subproblems simultaneously rather than sequentially, and aims to minimize total cost rather than individual cost components.

Recall that the joint production and transportation planning problem defined in Section 3 requires determining the following: production amount in each period, order delivery times and the number of both types of vehicles to be used for deliveries. If the order delivery dates are known, the production amounts and the vehicles used in each period can be determined optimally using Algorithms I and II, respectively. This structure of the joint problem enables us to define a solution by an array of size $N$, where the $i$ th element 
stores the information regarding the delivery period of order $i$. The tabu search begins with an initial seed solution in which each order's delivery time is set to its deadline. At each iteration, a neighbourhood of the current seed is generated and all solutions in the neighbourhood are evaluated for their costs. The cost of a solution is simply the summation of the optimal objective function values of the two subproblems. The solution with the least cost in the neighbourhood and that is not tabu is selected as the new seed, and a new iteration begins. The search for the best solution continues until the stopping criterion is met.

The neighbourhood of a seed is generated by changing the delivery dates of all orders one order at a time, keeping the delivery dates of the remaining orders as they stand at that point. In changing the delivery date of order $i$, we consider a feasible range of values, that is $\left[E_{i}, D_{i}\right] . E_{i}$ here represents the earliest feasible delivery date of order $i$. Its value is computed by taking into account the production capacity over time and the sizes of all orders that must be completed before $D_{i}$. Defining $\delta_{t, i}$ as the total size of all orders apart from order $i$ that must be completed in or before period $t$, we propose the following procedure to obtain values for $E_{i}$ for all $i$ :

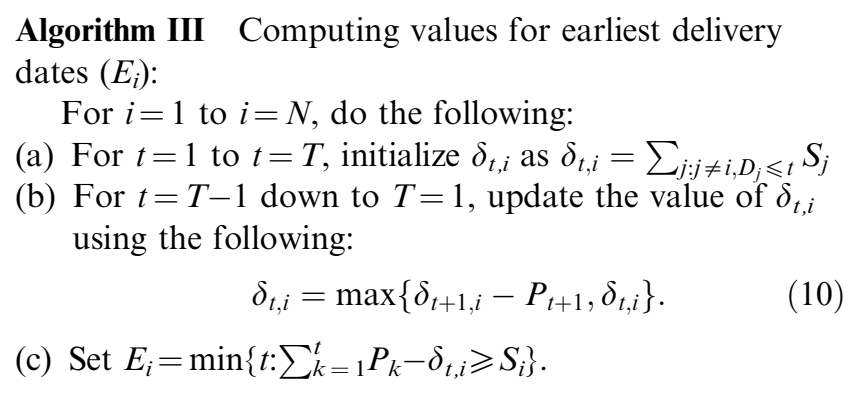

To explain why $E_{i}$, as found in the above algorithm, is the earliest delivery date for order $i$, let us start elaborating from the last step of the algorithm. $\sum_{k=1}^{t} P_{k}-\delta_{t, i}$ is the remaining of the total production capacity in periods $1, \ldots, t$ that can be reserved for order $i$. If $\sum_{k=1}^{t} P_{k}-\delta_{t, i}<S_{i}$ for some period $t$, then it is not possible to finish the production of order $i$ before or in period $t$. If order $i$ can be delivered before or in period $t$, then it must be true that $\sum_{k=1}^{t} P_{k}-\delta_{t, i} \geqslant S_{i}$, and therefore, in order to determine the earliest delivery date, we choose the smallest among all such $t$. The $\delta_{t, i}$ values for all $t$ are found in the first and second steps of the algorithm. Initially, $\delta_{t, i}$ is set to $\sum_{j: j \neq i, D_{j} \leqslant t} S_{j}$, that is, the total size of all orders other than $i$ with deadlines smaller than or equal to $t$. Thereafter, $\delta_{t, i}$ values are updated by tracing backwards from $t=T-1$ to all periods $T-2, \ldots, 1$. The update is done using Equation (10). In this equation, if the maximum is given by $\delta_{t+1, i}-P_{t+1}$, then, given that only $\delta_{t, i}$ units are produced within the first $t$ periods for orders other than $i$, the production capacity in period $t+1$ is not enough to make timely future deliveries. Therefore, the excess requirement (ie, $\left.\delta_{t+1, i}-P_{t+1}-\delta_{t, i}\right)$ also must be satisfied through the production in the first $t$ periods.

The job of which delivery date has been changed to form the newly selected seed at each iteration is added to the tabu list. Therefore, a solution in a neighbourhood is considered tabu if this solution is constructed by changing the delivery date of a job on the tabu list. However, we use the following rule as an aspiration criterion: If the best solution in the neighbourhood has less cost than that of the best solution so far, then it is taken as the new seed, even if it is tabu. In the next section, we present our numerical experimentation with the three solution approaches and the tabu search heuristic. As will be discussed in this section, we use varying tabu lengths for instances with different order sizes.

\section{Computational analysis}

We first report the results of a computational analysis to quantify the savings from the coordinated solution and to examine how the resulting costs of the three approaches differ under varying problem parameters. Thereafter, we present some results from a comparison of the tabu search heuristic with the coordinated solution, that is, the optimal solution of the Integrated Model. More specifically, we seek answers to the following questions:

- How do the inventory holding cost (ie, $H$ ) and the vehicle holding cost (ie, $W$ ) affect the outcomes of the three solution approaches? How do the results change with varying order sizes?

- How does the availability pattern of the type II vehicles affect the differences in costs? Here, we consider the average number of type II vehicles available in each period during the planning horizon and the degree of changes in their availability from one period to another.

- What is the impact of production capacity on the outcomes of the different solutions? How do the results change at varying levels of the inventory holding cost and the vehicle holding cost?

- What is the worst case and the average performance of the tabu search heuristic compared with the coordinated solution? How do these results change under varying problem parameters?

As discussed in Section 3, the coordinated solution leads to the optimal costs and the hierarchical solution is an improvement over the myopic solution. Therefore, it is true for any instance that $\operatorname{Cost}_{m} \geqslant \operatorname{Cost}_{h} \geqslant \operatorname{Cost}_{c}$. However, in light of the first three questions above, our objective is to examine the magnitudes of the differences 
between the cost values under relevant combinations of parameter settings. With this objective, we define the following measures for a problem instance:

$$
\begin{aligned}
\Delta_{m, h} & =\frac{\text { Cost }_{m}-\text { Cost }_{h}}{\text { Cost }_{m}} \times 100 \%, \\
\Delta_{h, c} & =\frac{\text { Cost }_{h}-\text { Cost }_{c}}{\text { Cost }_{h}} \times 100 \% \text { and } \\
\Delta_{m, c} & =\frac{\text { Cost }_{m}-\text { Cost }_{c}}{\text { Cost }_{m}} \times 100 \% .
\end{aligned}
$$

Note that each of the $\Delta_{m, h}, \Delta_{h, c}$ and $\Delta_{m, c}$ values refers to the percentage cost improvement of one solution approach over another. Given that the mathematical models for the production subproblem and the coordinated solution are solved optimally, we have $\Delta_{m, h} \geqslant 0, \Delta_{h, c} \geqslant 0$ and $\Delta_{m, c} \geqslant 0$. To test the performance of the heuristic, we consider how the resulting cost for an instance compares with the lower bound provided by GAMS. Before we proceed with a detailed discussion of these results, we first present the experimental design.

\subsection{Experimental design}

Considering the questions highlighted at the beginning of this section, we use the following six parameters as factors in our experiment: vehicle holding cost $(W)$, inventory holding cost $(H)$, production capacity, order sizes, average number of type II vehicles per period and period-to-period variability of type II vehicles. The length of the production planning horizon is taken as 1 month, and 1 day is considered as a period (ie, $T=30$ ). We do not take the length of the planning horizon, vehicle costs and capacities as factors of analysis, and therefore we keep their values fixed as $T=30, C_{1}=1000, C_{2}=100$ and $K=100$, respectively. In what follows, we describe the factor levels used in the experimentation and how they are generated.

Vehicle holding cost: We consider five levels for this factor and generate them around the value of $\beta$, which is the maximum number of periods that holding a vehicle is justified. It can be observed from Expression (9) that there exists a unique value of $\beta$ that corresponds to every value of $W$. Furthermore, the hierarchical solution explicitly utilizes this value. A commonly used value of $\beta$ by the industry practice that motivated this study is equal to 4 . Therefore, we take low, medium and high values of $\beta$ as 2 , 4 and 8 , respectively. As $\beta$ is an important parameter for the purposes of this study, our analysis also considers its extreme values, which are $\beta=0$ and $\beta=32$. The values of $W$ that correspond to the different levels of $\beta$ are reported in Table 2.

Inventory holding cost: Five levels of $H$ are generated around a factor that we refer to as $\alpha$ and define as follows:

$$
\alpha=\left\lceil\frac{C_{1}-C_{2}}{H \times K}\right\rceil-1 \text {. }
$$

In our setting, when an order is ready to be delivered, there clearly exists a trade-off between delivering it right away or holding it in the inventory so that a less costly delivery option available in a future period can be used. $\alpha$ shows the maximum number of periods that a full truckload of items can be stocked at the expense of inventory holding costs but where the savings in transportation costs exceed these extra costs. Expression (11) implies that there exists a unique value of $\alpha$ for each $H$. We consider 10, 4, 2, 1 and 0.25 as different levels of $H$, which correspond to $\alpha$ values of $0,2,4,8$ and 32 , respectively.

Production capacity: It is assumed that there are 6 working days followed by a no-production day. Therefore, there are 26 production periods within the planning horizon. Although there is no production during the remaining 4 days, costs are incurred for carrying inventories of items and inventories of vehicles over these periods. The production capacity over the production periods is constant. We consider two levels for production capacity: 1000 units/day and 1500 units/day. As will be discussed later, we generate the order sizes in such a way that the sizes of all orders to be produced sum up to 24000 units. This being said, the average load of the system, defined as total size of all orders/total production capacity, is approximately $90 \%$ in the low production-capacity case (ie, $24000 /(26 \times 1000)$, and is approximately $60 \%$ in the high production-capacity case (ie, $24000 /(26 \times 1500))$.

Order sizes: Three different sets of orders are used in combination with other factors. All orders in a set have small, medium or large sizes. An order's size is determined by how it compares with the vehicle capacity (ie, $K=100$ ) and with the low level of the daily production capacity (ie, $\left.P_{t}=1000\right)$. Small-sized orders have fewer than 100 items, medium-sized orders have more than 100 items but fewer than 1000 items, and large-sized orders have more than 1000 items. The number of items in a small order is taken as a uniformly distributed random variable between 10 and 100. The number of items in a medium order is generated from a uniform distribution ranging from 100 to 1000 . The sizes of orders in the third set are generated using a uniformly distributed random variable between 1000 and 4000. The total number of items over all orders in a set is kept at 24000 units. This sum is maintained by reducing the number of items in the first order that makes the total size greater than 24000 . As a result, for our experiment, the number of orders in the sets of small, medium and large orders turns out to be 450, 45 and 10, respectively.

Availability pattern of type II vehicles: The average number of type II vehicles per period and their period-toperiod variability determine the pattern of type II vehicle arrivals. These two attributes are taken as the factors of analysis and two levels are considered for each. The number of type II vehicles in each period is generated using a discrete uniform distribution, and the availability pattern of type II vehicles is controlled using the mean and the 
coefficient of variation $(\mathrm{CV})$ of this random variable. The average number of type II vehicles per day assumes either a value of 2.5 vehicles/day or 7.5 vehicles/day. The bounds of the uniformly distributed random variable corresponding to the number of type II vehicles per day are chosen in such a way that the CV is either 0.2 or 0.6 . The parameters of the uniformly distributed random variable used to create different availability patterns are reported in Table 1 . For example, an average number of 2.5 vehicles/day combined with 0.2 as the CV represents a case where type II vehicles are less available but arrive in a steady stream. Similarly, an average number of 7.5 vehicles/day combined with 0.6 as the CV represents a case where type II vehicles are more available in number, but their availability shows more variability among different days.

The factor levels used in the analysis and described above in detail are summarized in Table 2. In total, there are 600 different experimental settings. For each combination of factor levels, 10 random instances are solved.

\subsection{Comparison of the three solution approaches}

To compare the three solution approaches, we coded all the mathematical models discussed in Section 3 using GAMS version 22.6 (with CPLEX 11.0 as the solver) and run on a Linux box with $8 \mathrm{GBs}$ of physical memory, running Debian Lenny (5.0.7) on $8 \times$ Intel Xeon E5430 processors at $2.66 \mathrm{GHz}$. Recall from Theorem 1 that the production subproblem is NP-hard in the strong sense;

Table 1 Parameter settings for arrival patterns of type II vehicles

\begin{tabular}{lcc}
\hline \multirow{2}{*}{$\begin{array}{l}\text { Average number } \\
\text { of vehicles per day }\end{array}$} & \multicolumn{2}{c}{ Period-to-period variability } \\
\cline { 2 - 3 } & $C V=0.2$ & $C V=0.6$ \\
\hline Low $(2.5$ vehicles/day) & {$[2,3]$} & {$[0,5]$} \\
High (7.5 vehicles/day) & {$[5,10]$} & {$[0,15]$} \\
\hline
\end{tabular}

Table 2 Experimental design

\begin{tabular}{ll}
\hline Design parameter & \multicolumn{1}{c}{ Levels } \\
\hline Vehicle holding cost & $\beta=(0,2,4,8,32)$ or \\
& $W=(1000,400,200,100,25)$ \\
& $\alpha=(0,2,4,8,32)$ or \\
Inventory holding cost & $H=(10,4,2,1,0.25)$ \\
Production capacity & High $(1500)$, Low $(1000)$ \\
$\begin{array}{l}\text { Average \# of type II } \\
\text { vehicles per day }\end{array}$ & $C V=0.5)$, High $(7.5)$ \\
$\begin{array}{l}\text { Variability of the \# of } \\
\text { type II vehicles per day } \\
\text { Order sizes }\end{array}$ & Low $\sim \mathrm{U}(10,100)$, \\
& Medium $\sim \mathrm{U}(100,1000)$, \\
& High $\sim \mathrm{U}(1000,4000)$ \\
\hline
\end{tabular}

therefore, for practical problem sizes, these solutions may require extensive computational time. Thus, we have limited the solution time of each model for a problem instance to $36000 \mathrm{CPU}$ seconds. The model for the production subproblem was solved with a less than $0.02 \%$ optimality gap in 5950 out of 6000 instances and the Integrated Model was solved with a less than $0.11 \%$ optimality gap. For the remaining 50 instances, GAMS failed to provide a solution because of memory interruption. For these instances, we modified the GAMS model and accepted a $0.2 \%$ optimality gap as the termination criterion.

6.2.1. The effects of the inventory holding cost and the vehicle holding cost. In this section, we report our observations on how the inventory and vehicle holding costs affect the outcomes of the three solution approaches and how the results change with varying order sizes. For this analysis, we look into the averages of $\Delta_{m, h}, \Delta_{h, c}$ and $\Delta_{m, c}$ over all instances of the same-sized orders. The results for small, medium and large orders are summarized in Tables 3, 4 and 5, respectively. The values of $\alpha$ and $\beta$ change along the rows and columns of these tables. In each cell, the averages of $\Delta_{m, h}, \Delta_{h, c}$ and $\Delta_{m, c}$ over all instances with the corresponding $\alpha$ and $\beta$ values are noted. For example, the entries in the second row, second column of Table 3 show that over all instances with small orders, $\alpha=0$ and $\beta=0$, the averages of $\Delta_{m, h}, \Delta_{h, c}$ and $\Delta_{m, c}$ amount to $0.00 \%, 10.58 \%$ and $10.58 \%$, respectively.

It can be observed from Tables 3,4 and 5 that $\Delta_{m, h}=0$ when $\beta=0$. This result is because the first steps of the myopic solution and the hierarchical solution are the same,

Table 3 Average of $\Delta_{m, h}, \Delta_{h, c}$ and $\Delta_{m, c}$ values for small-sized orders

\begin{tabular}{rrrrrr}
\hline & $\beta=0$ & $\beta=2$ & $\beta=4$ & $\beta=8$ & $\beta=32$ \\
\hline$\alpha=0$ & $0.00 \%$ & $2.21 \%$ & $3.19 \%$ & $3.69 \%$ & $4.06 \%$ \\
& $10.58 \%$ & $8.60 \%$ & $7.73 \%$ & $7.49 \%$ & $7.68 \%$ \\
& $10.58 \%$ & $10.58 \%$ & $10.64 \%$ & $10.86 \%$ & $11.38 \%$ \\
$\alpha=2$ & $0.00 \%$ & $2.23 \%$ & $3.18 \%$ & $3.66 \%$ & $4.02 \%$ \\
& $11.28 \%$ & $9.30 \%$ & $8.42 \%$ & $7.97 \%$ & $7.84 \%$ \\
& $11.28 \%$ & $11.28 \%$ & $11.28 \%$ & $11.29 \%$ & $11.49 \%$ \\
$\alpha=4$ & & & & & \\
& $0.00 \%$ & $2.20 \%$ & $3.15 \%$ & $3.63 \%$ & $3.99 \%$ \\
& $11.45 \%$ & $9.50 \%$ & $8.63 \%$ & $8.18 \%$ & $7.87 \%$ \\
& $11.45 \%$ & $11.45 \%$ & $11.45 \%$ & $11.45 \%$ & $11.48 \%$ \\
$\alpha=8$ & & & & & \\
& $0.00 \%$ & $2.20 \%$ & $3.16 \%$ & $3.65 \%$ & $4.01 \%$ \\
& $11.43 \%$ & $9.48 \%$ & $8.60 \%$ & $8.14 \%$ & $7.79 \%$ \\
& $11.43 \%$ & $11.43 \%$ & $11.43 \%$ & $11.43 \%$ & $11.43 \%$ \\
$\alpha=32$ & & & & & \\
& $0.00 \%$ & $2.26 \%$ & $3.25 \%$ & $3.75 \%$ & $4.12 \%$ \\
& $11.52 \%$ & $9.52 \%$ & $8.60 \%$ & $8.13 \%$ & $7.77 \%$ \\
& $11.52 \%$ & $11.52 \%$ & $11.52 \%$ & $11.52 \%$ & $11.52 \%$ \\
\hline
\end{tabular}


Table 4 Average of $\Delta_{m, h}, \Delta_{h, c}$ and $\Delta_{m, c}$ values for medium-sized orders

\begin{tabular}{rrrrrr}
\hline & $\beta=0$ & $\beta=2$ & $\beta=4$ & $\beta=8$ & $\beta=32$ \\
\hline$\alpha=0$ & $0.00 \%$ & $5.91 \%$ & $10.42 \%$ & $13.75 \%$ & $16.39 \%$ \\
& $11.20 \%$ & $10.97 \%$ & $11.22 \%$ & $10.93 \%$ & $10.74 \%$ \\
& $11.20 \%$ & $15.97 \%$ & $20.04 \%$ & $22.66 \%$ & $24.94 \%$ \\
$\alpha=2$ & $0.00 \%$ & $6.12 \%$ & $10.74 \%$ & $14.13 \%$ & $16.77 \%$ \\
& $18.62 \%$ & $14.96 \%$ & $13.12 \%$ & $11.97 \%$ & $11.51 \%$ \\
& $18.62 \%$ & $19.78 \%$ & $21.88 \%$ & $23.79 \%$ & $25.68 \%$ \\
$\alpha=4$ & & & & & \\
& $0.00 \%$ & $6.20 \%$ & $10.75 \%$ & $14.03 \%$ & $16.54 \%$ \\
& $22.10 \%$ & $17.97 \%$ & $14.83 \%$ & $12.89 \%$ & $12.22 \%$ \\
$\alpha=8$ & $22.10 \%$ & $22.60 \%$ & $23.32 \%$ & $24.41 \%$ & $26.00 \%$ \\
& & & & & \\
& $0.00 \%$ & $6.12 \%$ & $10.74 \%$ & $14.13 \%$ & $16.77 \%$ \\
& $23.78 \%$ & $19.84 \%$ & $16.57 \%$ & $13.57 \%$ & $12.20 \%$ \\
$\alpha=32$ & $23.78 \%$ & $24.25 \%$ & $24.85 \%$ & $24.96 \%$ & $26.25 \%$ \\
& $0.00 \%$ & $6.11 \%$ & $10.75 \%$ & $14.02 \%$ & $16.53 \%$ \\
& $25.50 \%$ & $21.53 \%$ & $18.08 \%$ & $15.30 \%$ & $13.07 \%$ \\
& $25.50 \%$ & $25.74 \%$ & $25.91 \%$ & $26.23 \%$ & $26.70 \%$ \\
\hline
\end{tabular}

Table 5 Average of $\Delta_{m, h}, \Delta_{h, c}$ and $\Delta_{m, c}$ values for large-sized orders

\begin{tabular}{rrrrrr}
\hline & $\beta=0$ & $\beta=2$ & $\beta=4$ & $\beta=8$ & $\beta=32$ \\
\hline$\alpha=0$ & $0.00 \%$ & $6.90 \%$ & $13.51 \%$ & $18.23 \%$ & $22.11 \%$ \\
& $2.70 \%$ & $2.42 \%$ & $2.62 \%$ & $2.85 \%$ & $3.11 \%$ \\
& $2.70 \%$ & $9.07 \%$ & $15.64 \%$ & $20.34 \%$ & $24.22 \%$ \\
$\alpha=2$ & $0.00 \%$ & $9.47 \%$ & $18.55 \%$ & $24.88 \%$ & $30.06 \%$ \\
& $4.02 \%$ & $3.66 \%$ & $4.04 \%$ & $4.66 \%$ & $5.58 \%$ \\
& $4.02 \%$ & $12.65 \%$ & $21.60 \%$ & $27.98 \%$ & $33.32 \%$ \\
$\alpha=4$ & & & & & \\
& $0.00 \%$ & $10.92 \%$ & $21.41 \%$ & $28.70 \%$ & $34.62 \%$ \\
& $5.36 \%$ & $5.06 \%$ & $5.35 \%$ & $5.83 \%$ & $6.98 \%$ \\
$\alpha=8$ & $5.36 \%$ & $15.22 \%$ & $25.25 \%$ & $32.32 \%$ & $38.37 \%$ \\
& $0.00 \%$ & $11.79 \%$ & $23.12 \%$ & $31.02 \%$ & $37.43 \%$ \\
& $6.74 \%$ & $6.55 \%$ & $6.76 \%$ & $7.08 \%$ & $8.20 \%$ \\
$\alpha=32$ & $6.74 \%$ & $17.28 \%$ & $27.83 \%$ & $35.24 \%$ & $41.61 \%$ \\
& $0.00 \%$ & $12.60 \%$ & $24.81 \%$ & $33.37 \%$ & $40.30 \%$ \\
& $8.59 \%$ & $8.54 \%$ & $8.52 \%$ & $8.48 \%$ & $9.23 \%$ \\
& $8.59 \%$ & $19.70 \%$ & $30.57 \%$ & $38.11 \%$ & $44.59 \%$ \\
\hline
\end{tabular}

but the hierarchical solution entails type II vehicles being carried to future periods as long as the savings justify the increase in vehicle holding costs. In the case of $\beta=0$, it is less costly to use a type I vehicle in any period rather than carry a type II vehicle from an earlier period. Therefore, the hierarchical solution reduces to the myopic solution, and hence $\Delta_{m, h}=0$.

Examining Tables 3, 4 and 5, we observe that the maximum of the average $\Delta_{m, h}$ values is $4.12 \%, 16.77 \%$ and $40.30 \%$ for the small, medium and large orders, respectively. These values are realized when $\beta$ attains its highest value. Excluding the values when $\beta=0$, the minimums are $2.20 \%, 5.91 \%$ and $6.90 \%$, and these values are realized when $\beta=2$. Furthermore, the average $\Delta_{m, h}$ values increase as $\beta$ increases in each row of Tables 3, 4 and 5. This implies that as it becomes less costly to carry type II vehicles over periods, the hierarchical solution uses this opportunity to reduce the costs of the myopic solution, and the potential of improvement is highest when the order sizes are largest.

The maximum of the average $\Delta_{h, c}$ values is $11.52 \%$, $25.50 \%$ and $9.23 \%$ for the small, medium and large orders, respectively. These values are realized when $\alpha$ attains its highest value. The minimums are $7.49 \%, 10.74 \%$ and $2.42 \%$, and they coincide with the cases where $\alpha=0$. Furthermore, the average $\Delta_{h, c}$ values increase as $\alpha$ increases in each row of Tables 4 and 5, and in most rows of Table 3 . This implies that the performance of the hierarchical solution approaches that of the coordinated solution as the inventory holding cost rate, $H$, increases. This result is because at high values of $H$, transportation costs constitute a lesser portion of total costs, and just by solving the production subproblem optimally (as in the hierarchical solution), the most substantial advances in minimizing total costs can be made. It is worthwhile to note that Table 3 exhibits some exceptions. For example, average $\Delta_{h, c}$ is $9.50 \%$ when $\alpha=4$ and $\beta=2$, whereas it is equal to $9.48 \%$ when $\alpha=8$ and $\beta=2$. We believe this result is because of the fact that inventory holding costs constitute a lesser portion of total costs compared with transportation costs for small orders. Therefore, average $\Delta_{h, c}$ is not very sensitive to changes in $\alpha$, and hence these exceptions are not representative of the general behaviour.

Tables 3, 4 and 5 suggest that the maximum of the average $\Delta_{m, c}$ values is $11.52 \%, 26.70 \%$ and $44.59 \%$ for small, medium and large orders, respectively. These values are realized when $\alpha$ and $\beta$ are at their highest values. The minimums are $10.58 \%, 11.20 \%$ and $2.70 \%$, and these values are realized when $\alpha$ and $\beta$ assume their smallest values. Furthermore, average $\Delta_{m, c}$ values are non-decreasing in $\beta$ in all order sizes, and increasing in $\alpha$ when orders are medium or large sized. As seen in Table 3 , the values are predominantly increasing in $\alpha$ when orders are small, but there are some exceptions. We again attribute this result to the fact that the amount of inventory held is lower for small orders, and therefore the behaviour of average $\Delta_{m, c}$ with respect to $\alpha$ is not well observed. As a result of these observations, we conclude that the savings because of the coordinated solution are in fact significant, and the percentage savings over the myopic solution increases as inventory and vehicle holding costs become smaller. Our reasoning for why $\Delta_{m, c}$ decreases with an increasing $H$ is similar to the reason that we propose for why $\Delta_{h, c}$ follows the same pattern. Recall that in the myopic and hierarchical solutions, the production subproblem is solved optimally. On the other hand, we think $\Delta_{m, c}$ 
decreases with an increasing vehicle holding cost for the following reason: In this case, carrying type II vehicles over periods becomes cost-ineffective, and the coordinated solution approaches an output where no vehicles are carried over periods and type I vehicles are used only if they are needed in addition to the number of type II vehicles that become available in a period. Notice that this is basically the output of the myopic solution for the transportation subproblem.

6.2.2. The effects of the availability pattern of type II vehicles. In this section, we discuss the results of our computational study within the context of the second objective, that is, to determine how the availability pattern of type II vehicles affects cost differences. For this purpose, we look into the averages of $\Delta_{m, h}, \Delta_{h, c}$ and $\Delta_{m, c}$ over all instances with the same arrival pattern. Recall that the arrival pattern of type II vehicles is identified by two attributes, the mean and the variability of the number of type II vehicles per day. The results for four different availability patterns are summarized in Table 6 . The values of the two attributes change along the rows and columns of these tables. In each cell, the averages of $\Delta_{m, h}, \Delta_{h, c}$ and $\Delta_{m, c}$ over all instances with the same availability pattern of type II vehicles are noted.

It can be observed from Table 6 that percentage improvements of both the hierarchical solution and the coordinated solution over the myopic solution, as represented by $\Delta_{m, h}$ and $\Delta_{m, c}$, respectively, increase with an increase in the average number of type II vehicles available. This result implies that the value of coordination is higher when the opportunity of savings because of effective utilization of the different transportation options is higher. Observe also that $\Delta_{h, c}=2.02 \%$ when $C V=0.2$, and $\Delta_{h, c}=2.97 \%$ when $C V=0.6$. This suggests that although the opportunity of savings is limited at low levels of the average number of type II vehicles per day, the hierarchical solution performs almost as well as the coordinated solution in capturing this opportunity. When the results

Table 6 Average of $\Delta_{m, h}, \Delta_{h, c}$ and $\Delta_{m, c}$ values under different arrival patterns of type II vehicles

\begin{tabular}{lcc}
\hline Average \# of vehicles/day & \multicolumn{2}{c}{ Day-to-day variability } \\
\cline { 2 - 3 } & $C V=0.2$ & $C V=0.6$ \\
\hline Low (2.5 vehicles/day) & $4.26 \%$ & $4.81 \%$ \\
& $2.02 \%$ & $2.97 \%$ \\
& $6.23 \%$ & $7.68 \%$ \\
High (7.5 vehicles/day) & $14.90 \%$ & $16.38 \%$ \\
& $15.38 \%$ & $19.46 \%$ \\
& $28.40 \%$ & $33.12 \%$ \\
\hline
\end{tabular}

Table 7 Average of $\Delta_{m, h}, \Delta_{h, c}$ and $\Delta_{m, c}$ values at varying levels of production capacity

\begin{tabular}{lc}
\hline \multicolumn{3}{c}{ Production capacity } \\
\hline Low $(\%)$ & High $(\%)$ \\
\hline 10.51 & 9.66 \\
4.59 & 15.33 \\
14.64 & 23.07 \\
\hline
\end{tabular}

in Table 6 are examined for the variability in number of type II vehicle arrivals, we observe that the value of coordination becomes higher as the dispersion increases. In addition, the discrepancy between the performances of the coordinated solution and the hierarchical solution grows with increased variability.

6.2.3. Effects of production capacity. To determine the effects of production capacity on the performance of the three solution approaches, we investigate how the averages of $\Delta_{m, h}, \Delta_{h, c}$ and $\Delta_{m, c}$ change at different production levels. The results are summarized in Table 7 .

Recall that we consider average $\Delta_{m, h}$ as a measure to quantify the savings that can be achieved by optimal usage of transportation availabilities when production and transportation decisions are made sequentially. Table 7 shows that when production capacity is increased from its low level to its high level, $\Delta_{m, h}$ decreases slightly, implying that a less-stringent constraint on production capacity does not particularly improve the hierarchical solution's transportation decisions. This is because the production capacity has a foremost impact on the production subproblem and an increasing production capacity helps reduce inventory holding costs in both the myopic and the hierarchical solutions; however, depending on the time-varying availability of type II vehicles, a different output of the production subproblem because of changes in production capacity may either decrease or increase transportation costs for both solutions. On the other hand, $\Delta_{h, c}$ and $\Delta_{m, c}$ increase significantly at the high level of the production capacity. This result shows that integrating production and transportation decisions helps take better advantage of resources (in particular, increased production capacity) to decrease total cost.

6.2.4. Performance of the tabu search heuristic. In light of the fourth question of interest, we test the performance of the heuristic with respect to the coordinated solution using the 6000 instances described in Section 6.1. As the Integrated Model for obtaining the coordinated solution cannot be solved optimally for all instances, we compare the cost of the heuristic solution with the lower bound 
provided by GAMS. In obtaining heuristic solutions for instances with low, medium and high order sizes, we set the tabu length as 200, 25 and 7, respectively. We also use the following scheme for terminating the search: If the algorithm fails to improve the best solution for 2000 consecutive iterations, the seed is replaced with the best solution so far, but the tabu list is not changed. The algorithm is terminated if this happens 100 times or if the total search time exceeds 60 CPUs.

As a result of our experimentation, we observed that the tabu search performs quite well in general. In more than $37 \%$ of the instances (2256 out of 6000 ), the tabu search terminated with an optimal solution. In approximately $90 \%$ of the instances (5421 out of 6000 ), the deviation between the cost of the heuristic solution and the lower bound was as much as $1 \%$ of the lower bound, and in approximately $99.7 \%$ of the instances (5983 out of 6000), the deviation was at most $5 \%$ of the lower bound. The average and the maximum percentage deviations were $0.31 \%$ and $10.13 \%$, respectively. The maximum deviation was realized at an instance where the vehicle holding cost is high (ie, $\beta=0$ ), the inventory holding cost is high (ie, $\alpha=0$ ), production capacity is low, orders are of medium size and the number of type II vehicles per day is high on average but shows variability among different days. In fact, after a detailed analysis of the results, we observe that only two of the parameters have an impact worth noting on the performance of the heuristic: order size and variability in the number of type II vehicles. As Table 8 shows, the average deviation of the heuristic from the lower bounds is most when orders are of medium size, and the mean and the variability of the number of type II vehicles are high. Recall that the proposed tabu search is based on optimally solving the production and transportation subproblems for different delivery dates of orders at consecutive iterations. Although the maximum average percentage deviation (ie, $1.62 \%$ ) is still very small, the reason why this value occurs on medium-size orders and with high mean and variability of the number of type II vehicles, may be because of the mechanism of the tabu search. More specifically, when the orders are of extreme sizes (either small or large), there are few alternative combinations of delivery dates and it is more likely that the true combination is considered within the tabu search. However, if the orders are of medium size, there may be many combinations of delivery dates and the impact of a suboptimal combination may be amplified when the number of type II vehicles has high mean and variability.

\section{Extensions and conclusions}

In this paper, we studied a manufacturer's joint production and transportation planning problem, specifically, to schedule orders with deadlines to minimize the sum of inventory holding and outbound transportation costs without allowing any tardiness. An important characteristic of the problem setting is that there are two vehicle types; one of unlimited availability but high cost, and the other of limited and time-varying availability but lower cost. As Chen (2010) reports, there are few papers on integrated production and outbound scheduling that consider heterogenous vehicles. In all these papers, heterogeneity is due to differences in delivery speed and cost. One contribution of our study is that we model the existence of heterogenous vehicles that differ in their time-varying availability and cost. As companies are increasingly relying on the services of multiple carriers to outsource their freight, we believe modelling this type of vehicle heterogeneity will find many applications and is also worthy of investigation in other problems settings.

Motivated by our observations from several industry practices, we have presented three approaches to solve the manufacturer's production and transportation planning problem in our setting: the myopic solution, the hierarchical solution and the coordinated solution. These approaches rely on solving two underlying subproblems (production and transportation), which were identified and mathematically formulated in the paper. The myopic solution and the hierarchical solution are based on solving the production subproblem first, followed by the transportation subproblem. Their difference is that, given the production decisions, the transportation subproblem is solved optimally in the hierarchical solution. The coordinated solution, on the other hand, aims to minimize total cost by making all related decisions in an integrated manner.

The problem of making production and transportation decisions in an integrated manner, however, as in the

Table 8 Average percentage deviation of the heuristic from the lower bounds, under different arrival patterns of type II vehicles and order sizes

\begin{tabular}{|c|c|c|c|c|c|c|}
\hline \multirow[t]{2}{*}{ Average \# of vehicles/day } & \multicolumn{2}{|c|}{$\begin{array}{c}\text { Small-sized orders } \\
\text { Day-to-day variability }\end{array}$} & \multicolumn{2}{|c|}{$\begin{array}{l}\text { Medium-sized orders } \\
\text { Day-to-day variability }\end{array}$} & \multicolumn{2}{|c|}{$\begin{array}{c}\text { Large-sized orders } \\
\text { Day-to-day variability }\end{array}$} \\
\hline & $C V=0.2$ & $C V=0.6$ & $C V=0.2$ & $C V=0.6$ & $C V=0.2$ & $C V=0.6$ \\
\hline Low $(\%)$ & 0.03 & 0.07 & 0.08 & 0.11 & 0.04 & 0.05 \\
\hline High (\%) & 0.22 & 0.32 & 0.94 & 1.62 & 0.15 & 0.15 \\
\hline
\end{tabular}


coordinated solution, is NP-hard in the strong sense. We show that the production subproblem has similar complexity. However, given the order delivery dates, we provide polynomial algorithms for solving the two subproblems. On the basis of these algorithms, we propose a tabu search heuristic for minimizing the total cost. The results of an extensive numerical analysis reveal that the heuristic takes less than a minute to find a solution, which deviates from the lower bound by at most $10.13 \%$ and by $0.31 \%$ on the average.

We also numerically compare the three solution approaches and provide several insights about how they differ under varying problem parameters. Recall that the myopic and the hierarchical solutions use the same input (the optimal solution of the production subproblem); however, the hierarchical solution explicitly considers the transportation availabilities to optimize the shipment costs. On the other hand, in the myopic solution, transportation decisions are made in a short-sighted manner, often with the sole objective of complying with an optimal production plan. Therefore, a detailed comparison of these two solution approaches helps us quantify the savings that can be achieved by explicit consideration of transportation costs and availabilities. In fact, our numerical results reveal that the maximum savings could amount to $71 \%$ compared with the cost of the myopic solution. On the average, the percentage cost improvement due to the hierarchical solution over the myopic solution is $10.08 \%$. A similar comparison between the hierarchical and coordinated solutions provides useful insights about the value of integrating transportation and production planning decisions; doing so can decrease total cost by as much as $58 \%$ compared with the cost of the hierarchical solution. The average percentage cost improvement because of the coordinated solution over the hierarchical solution is $9.97 \%$. Finally, comparing the myopic and coordinated solutions highlights the impact of optimally making transportation and production planning decisions and in an integrated manner. The maximum savings in this case can amount to $75 \%$ of the costs that occur when transportation planning is made non-optimally and following production decisions, that is, using the myopic solution. On the average, the percentage cost improvement due to the coordinated solution over the myopic solution is $18.9 \%$.

As a result of our numerical experimentation, we also arrive at several findings related to the questions highlighted at the beginning of Section 6. These findings, discussed in detail in the same section, suggest overall that the myopic solution performs close to the hierarchical solution as the type II-vehicle holding cost increases, and that the value of integration is particularly high when orders have large sizes, inventory and vehicle holding costs are low and the availability of the lower-cost vehicle shows high variability.

In our study, we considered a scenario in which different orders cannot be consolidated in the same vehicle and orders cannot be shipped in partial deliveries over time. Several extensions of this study can be developed considering different delivery characteristics. We would like to note that our analysis can easily be extended to allow for consolidation by slightly modifying Expression (4) of the Integrated Model and the second constraint of the transportation subproblem.

\section{References}

Bard JF and Nananukul N (2009). The integrated productioninventory-distribution-routing problem. Journal of Scheduling 12(3): 257-280.

Bard JF and Nananukul N (2010). A branch-and-price algorithm for an integrated production and inventory routing problem. Computers \& Operations Research 37(12): 2202-2217.

Chandra P and Fisher ML (1994). Coordination of production and distribution planning. European Journal of Operational Research 72(3): 503-517.

Chang Y-C and Lee C-Y (2004). Machine scheduling with job delivery coordination. European Journal of Operational Research 158(2): 470-487.

Chen B and Lee C-Y (2008). Logistics scheduling with batching and transportation. European Journal of Operational Research 189(3): 871-876.

Chen Z-L (2010). Integrated production and outbound distribution scheduling: Review and extensions. Operations Research 58(1): 130-148.

Chen Z-L and Pundoor G (2006). Order assignment and scheduling in a supply chain. Operations Research 54(3): 555-572.

Chen Z-L and Vairaktarakis GL (2005). Integrated scheduling of production and distribution operations. Management Science 51(4): 614-628.

Dawande M, Geismar HN, Hall NG and Sriskandarajah C (2006). Supply chain scheduling: Distribution systems. Production and Operations Management 15(2): 243-261.

Geismar HN, Laporte G, Lei L and Sriskandarajah C (2008). The integrated production and transportation scheduling problem for a product with a short lifespan. INFORMS Journal on Computing 20(1): 21-33.

Geismar HN, Dawande M and Sriskandarajah C (2011). Poolpoint distribution of zero-inventory products. Production and Operations Management 20(5): 737-753.

Hall NG and Potts CN (2003). Supply chain scheduling: Batching and delivery. Operations Research 51(4): 566-584.

Koç U, Toptal A and Sabuncuoglu I (2013). A class of joint production and transportation planning problems under different delivery policies. Operations Research Letters 41(1): 54-60.

Lee HL, So KC and Tang CS (2000). The value of information sharing in a two-level supply chain. Management Science 46(5): 626-643.

Lei L, Liu S, Ruszczynski A and Park S (2006). On the integrated production, inventory, and distribution routing problem. IIE Transactions 38(11): 955-970.

Li L (2002). Information sharing in a supply chain with horizontal competition. Management Science 48(9): 1196-1212.

Li C-L and Ou J (2005). Machine scheduling with pickup and delivery. Naval Research Logistics 52(7): 616-630.

Li C-L, Vairaktarakis GL and Lee C-Y (2005). Machine scheduling with deliveries to multiple customer locations. European Journal of Operational Research 164(1): 39-51. 
Li C-L and Vairaktarakis GL (2007). Coordinating production and distribution of jobs with bundling operations. IIE Transactions 39(2): 203-215.

Pundoor G and Chen Z-L (2005). Scheduling a productiondistribution system to optimize the tradeoff between delivery tardiness and distribution cost. Naval Research Logistics 52(6): 571-588.

Sarmiento AM and Nagi R (1999). A review of integrated analysis of production-distribution systems. IIE Transactions 31(11): 1061-1074.

Stecke KE and Zhao X (2007). Production and transportation integration for a make-to-order manufacturing company with a commit-to-delivery business mode. Manufacturing \& Service Operations Management 9(2): 206-224.
Wang H and Lee C-Y (2005). Production and transport logistics scheduling with two transport mode choices. Naval Research Logistics 52(8): 796-809.

Wang X and Cheng TCE (2007). Machine scheduling with an availability constraint and job delivery coordination. Naval Research Logistics 54(1): 11-20.

Wang X and Cheng TCE (2009). Production scheduling with supply and delivery considerations to minimize the makespan. European Journal of Operational Research 194(3): 743-752.

Zhong W, Dósa G and Tan Z (2007). On the machine scheduling problem with job delivery coordination. European Journal of Operational Research 182(3): 1057-1072.

Received May 2012; accepted December 2012 after two revisions 